\title{
Designing of a comprehensive high altitude EAS array for primary particle determination within the PAMIR-XXI project
}

\author{
N.P. Topchiev* \\ Lebedev Physical Institute of the Russian Academy of Sciences, Moscow, Russia \\ E-mail: topchievesci.lebedev.ru
}

\author{
A.S. Borisov \\ Lebedev Physical Institute of the Russian Academy of Sciences, Moscow, Russia \\ E-mail: asborisov55@mail.ru
}

\author{
V.V. Batraev, R. Bakhromzod, V.I. Galkin, A.R. Muqumov \\ Lomonosov Moscow State University, Faculty of Physics, Moscow, Russia
}

\begin{abstract}
The method is described for estimation of the parameters of primary particles of extensive air showers by a complex high mountain detector array, developed in the framework of PAMIR-XXI project. The results can be useful for other high altitude projects as well as for the method of EAS study in general. We present particular configurations of optical and charged particles detectors, methods of data processing, the achievable accuracy of reconstruction of primary particle parameters, namely, energy, direction, and mass/type. The most part of the results relates to the optical detectors which are able to deal with the EAS from nuclei in the range of primary energies $E_{0}=100 \mathrm{TeV}-100 \mathrm{PeV}$ and with the gamma-ray showers of energies $E_{\gamma} \geq 30 \mathrm{TeV}$. We also considered the options of the charged particle detector grids intended to estimate the direction and energy of the EAS in the range of $E_{0}=1 \mathrm{PeV}-1 \mathrm{EeV}$. The estimates of the accuracies should be considered as upper limits of real experimental accuracies.
\end{abstract}

35th International Cosmic Ray Conference - ICRC2017

10-20 July, 2017

Bexco, Busan, Korea

* Speaker. 


\section{Introduction}

Characteristics of an extensive air shower (EAS) at mountain altitudes considerably differ from those at sea level that must be taken into account when constructing the methods of estimation of the parameters of the primary particle. This is especially important if the aim is to improve the existing methods of registration or even to develop a new, based on a detailed simulation of the EAS development and the use of detectors of different types.

Such a problem was posed in the framework of the project "Pamir-XXI" [1] which implies the construction of a complex detector array of a new generation in the mountains of the Eastern Pamirs at the altitude of $\sim 4250 \mathrm{~m}$ above sea level. Today the perspective for the implementation of the project is not clear but the calculations and methodical work continue. The results can be useful for other Alpine projects and the method for EAS detection in general.

The Pamir-XXI project was planned as a comprehensive study of the primary cosmic rays (PCR) in a wide energy range $30 \mathrm{TeV}-1 \mathrm{EeV}$ as well as characteristics of nuclear interactions in the forward kinematic region which are not available for study at accelerators. It was supposed to use a network of charged particle detectors, hadron calorimeter and optical detectors for registering various components of EAS: electron-photon, muon, hadron and Cherenkov light (CL).

The proposed configurations of the optical part of the set-up and a charged particle detector network, as well as the ways of data handling do provide fundamentally smaller uncertainties in estimating the energy, direction and type of primary particles than traditional methods.

\section{Statement of the problem and the solution logic}

In order to succeed in the above mentioned studies it is enough to measure primary particle energy $E_{0}$, type (mass), arrival direction $\vec{\theta}$ and core location $\vec{x}_{\text {core }}$ at the observation level. These parameters are usually considered to be equally demanding with regard to the amount of experimental data used while making the parameter estimates. Analysis of the results of the work of many EAS set-ups since the middle of last century to the present day shows that this is not so: while it is possible to evaluate the energy and direction of the primary particle it turns out that it is impossible to estimate the primary mass. Practically, this means that even an approximate knowledge of the mass of the primary nucleus requires a much larger amount of experimental information per event.

That is why we insist that a detector array and data processing methods should be optimized with respect to the informatively most exacting problems, namely, the primary mass estimation and the $\gamma$-event selection.

For the measurements to be as consistent as possible one must consider the most informationrich component to be the reference component for all other measurables. Among the EAS components the Cherenkov light (CL) is definitely the most informative and makes it possible to solve all stated problems within the energy range mentioned even with a small duty cycle $(\leq 10 \%)$.

Taking into account these statements, one is forced, first of all, to construct an array of Cherenkov detectors optimized for the solution of primary type/mass estimation problem. The array should analyze both Cherenkov pulses (spatial-temporal distribution of CL) and Cherenkov [angular] images (CL spatial-angular distribution) and thus will include a network of widely spaced fast optical detectors and a set of a few optical telescopes. 
A network of charged particle detectors will be used at $E_{0}>1 \mathrm{PeV}$ for evaluation of $E_{0}, \vec{\theta}$, $\vec{x}_{\text {core }}$ to help the central calorimeter and muon detectors (presumably trackers) to estimate the primary mass.

The method for the primary mass estimation by charged particle data (when finally established) can be tuned with the help of the respective Cherenkov light method.

\section{Artificial events}

\subsection{Generation of showers}

Artificial events for the observation level $4250 \mathrm{~m}$ a.s.l. were generated with CORSIKA6.990 /QGSJET01 and CORSIKA7.5600/QGSJET-II-04 [2] for a set of fixed $E_{0}$ from $30 \mathrm{TeV}$ to 100 $\mathrm{PeV}$ and different primary types: $\mathrm{p}, \mathrm{He}, \mathrm{N}, \mathrm{S}, \mathrm{Fe}, \gamma$. CL spatial-temporal and spatial-angular distributions and CORSIKA standard particle files were stored.

\subsection{Processing of CL and particle detector data}

To process the spatial-temporal data of simulated events simple polynomial models were used for $\mathrm{CL}$ and particle lateral distributions $\mathrm{LD}(\mathrm{R})$ and fronts $\tau(\mathrm{R})$ :

$$
F_{L D}(R)=a_{0} /\left(1+a_{1} R+a_{2} R^{2}+a_{3} R^{3}\right), \quad \tau(R)=R\left(a_{0}+a_{1} R+a_{2} R^{2}\right) .
$$

The shower direction definition is done simultaneously with the core location search by fitting the measured front arrival times by the front model and the measured number of photons (photoelectrons)/particles by the lateral distribution model. During this fit $\tau(R)$ and $F_{L D}(R)$ parameters are also varied so that we obtain the best front and lateral distribution approximations for each event. $F_{L D}(R)$ is further integrated within a circle of a certain radius to form an estimate of $E_{0}$.

Processing of CL angular images does not require any model functions, it comprises the location of a spot's long axis and integration over certain regions of field of view (FOV). The primary direction estimate by spatial-temporal data is used.

\section{Optical part of Pamir-XXI detector array}

The optical part of the detector array uses spatial-temporal and spatial-angular distributions of CL for the estimation of primary particle parameters and, thus, must incorporate two sets of detectors (Fig. 1).

\subsection{Multiple use of event Cherenkov data}

Event sample volumes vary from 200 for $30-100 \mathrm{TeV}$ primaries to 30 for $100 \mathrm{PeV}$ primaries but CL data of each event includes:

- a $600 \times 600 \times 302$ histogram of spatial-temporal distribution covering a $600 \mathrm{~m} \times 600 \mathrm{~m}$ carpet and $\sim 300$ ns delay interval and

- a $250 \times 250 \times 108 \times 108$ histogram of spatial-angular distribution within a $500 \mathrm{~m} \times 500 \mathrm{~m}$ square of observation level with every $2 m \times 2 m$ bin imitating an imaging telescope of $27^{\circ} \times 27^{\circ}$ field of view. 
Pamir-XXI optical

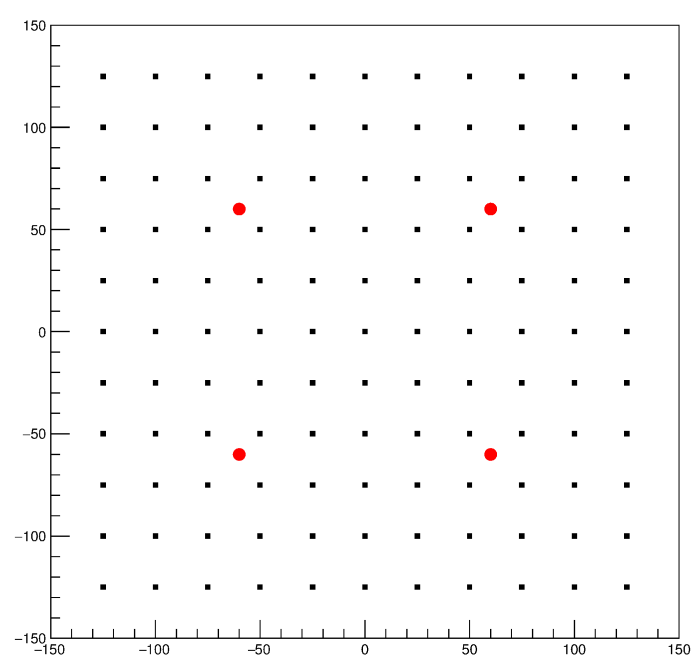

Figure 1: Optical detector array layout used in simulations: 1) rectangular network of $11 \times 11$ wide angle fast optical detectors of area $\sim 1 \mathrm{~m}^{2}$ and aperture $\sim 1 \mathrm{sr}$ set with $25 \mathrm{~m}$ step (squares), which must be able to determine the shower direction with an accuracy of at least $0.1^{\circ}$, core location with an accuracy of $\sim 1 \mathrm{~m}$ and the primary energy within $15 \%$ accuracy and also makes it possible to analyse the CL pulse shape; 2) optical image telescopes (circles) with mirrors of area $\sim 4 m^{2}$, field of view diameter of about $30^{\circ}$ and pixel diameter of $\sim 0.8^{\circ}$ spaced by $\sim 100 \mathrm{~m}$, which should enable the analysis of the CL angular distribution close to the shower core and be sensitive to the primary particle mass.

Thus, each event can be used many times by choosing different $1 \mathrm{~m} \times 1 \mathrm{~m}$ bins of the 1 st histogram as detectors of fast network and different $2 m \times 2 m$ bins of the 2 nd histogram as imaging telescopes.

\subsection{CL spatial-temporal distribution processing results}

Uncertainties of core location and primary direction determination by fast optical detector network meet the above set requirements $\left(\sim 1 \mathrm{~m}\right.$ and $\left.<0.1^{\circ}\right)$. The results for pure CL signal in photons and signal + night sky background (BG) $\left(10^{8} \mathrm{~cm}^{-2} \mathrm{sr}^{-1} \mathrm{~s}^{-1}\right)$ in photoelectrons are almost the same for $E_{0} \geq 1 \mathrm{PeV}$. The situation changes for primary energies $30-100 \mathrm{TeV}$ but the uncertainties still lie within the pre-set limits which gives hope to detect gamma-events in this energy range (Table 1).

Table 1: Core location, $m$, and primary direction, ${ }^{\circ}$, uncertainties

\begin{tabular}{lllll}
\hline event/signal & core loc.mean & core loc.RMS & prim.dir.mean & prim.dir.RMS \\
\hline $60 \mathrm{TeV}$ p, CL & 1.6 & 0.93 & 0.044 & 0.033 \\
$60 \mathrm{TeV}$ p, CL+BG & 2.6 & 1.9 & 0.076 & 0.064 \\
$60 \mathrm{TeV}$ p, CL+BG, $>$ 200phel & 2.4 & 1.6 & 0.059 & 0.048 \\
\hline $30 \mathrm{TeV} \gamma, \mathrm{CL}$ & 1.0 & 0.59 & 0.030 & 0.020 \\
$30 \mathrm{TeV} \gamma, \mathrm{CL}+\mathrm{BG}$ & 1.8 & 1.3 & 0.058 & 0.052 \\
$30 \mathrm{TeV} \gamma, \mathrm{CL}+\mathrm{BG},>$ 200phel & 1.5 & 1.1 & 0.051 & 0.046 \\
\hline
\end{tabular}

Table 2: CL300 relative fluctuations for $E_{0} \geq 1 \mathrm{PeV}, \%$

\begin{tabular}{llll}
\hline$E_{0}, \mathrm{PeV}$ & proton & nitrogen nucleus & iron nucleus \\
\hline 1 & 15 & 10 & 8 \\
10 & 14 & 8 & 5 \\
100 & 13 & 7 & 5 \\
\hline
\end{tabular}


Table 3: CL300 relative fluctuations for $E_{0}=30-100 \mathrm{TeV}, \%$

\begin{tabular}{llll}
\hline$E_{0}, \mathrm{TeV}$, prim.patricle & $\mathrm{CL}$ photons & $\mathrm{CL}+\mathrm{BG}$, photoelectrons & $\mathrm{CL}+\mathrm{BG},>$ 200phel \\
\hline 60, proton & 18 & 20 & 18 \\
100, proton & 13 & 16 & 16 \\
\hline $30, \gamma$ & 5 & 10 & 9 \\
$50, \gamma$ & 2 & 9 & 8 \\
\hline
\end{tabular}

The primary energy $E_{0}$ is estimated as an integral $C L 300=\int_{0}^{300} R d R F_{L D}(R)$ of CL lateral distribution (LD) over a circle of radius $300 \mathrm{~m}$ centered at the axis. Parameters of LD are obtained as a result of a joint fit of CL LD and front models to CL spatial-temporal data of individual EAS. Uncertainties of CL300 for $E_{0} \geq 1 \mathrm{PeV}$ are indifferent to night sky BG and conversion to photoelectrons (Table 2). The situation is different for the 30-100 TeV range: mixing of CL and BG photons and their conversion to photoelectrons increase the uncertainties (Table 3). Introduction of a threshold (e.g., 200 photoelectrons) for each fast detector somewhat reduces the uncertainties.

\subsection{CL spatial-angular distribution processing results}

The method for processing of CL angular images uses the spot shape parameters that provide maximum separation of showers initiated by primaries of different types. We calculate the integrals $S_{i}, i=1,2,3,4$ over rectangular segments of the FOV in the vicinity of the CL spot and use their ratios $r_{i j}=S_{i} / S_{j}$ as features characterizing the events. Maps for $0.75^{\circ} \times 0.75^{\circ}$ pixel size are used because this resolution turned out to be the most effective in preliminary studies [1]. Figure 2 gives the geometry of the image processing.

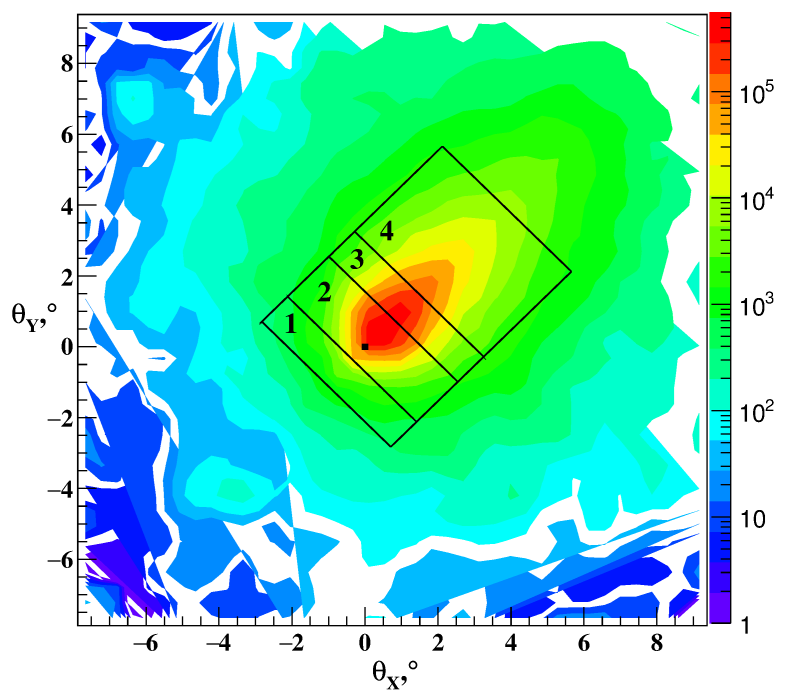

Figure 2: Shower image and rectangular areas of integration aligned along the spot long axis. Black square in the center of FOV marks the shower arrival direction. CL spot is sliced by rectangles 1,2,3,4 across its long axis. A stripe formed by the rectangles is $5^{\circ}$ wide. The outer side of rectangle 1 is placed at $-1.5^{\circ}$ with respect to the arrival direction. The image is integrated within the rectangles, integrals $S_{i}$ form ratios $r_{i j}$. The widths of the rectangles are varied so that to find $r_{i j}$ giving the best separation of images produced by different primary particles.

Optimum selection criteria have been found for $\mathrm{p}-\mathrm{N}$ and N-Fe pairs (QGSJET01) at 1, 10 and $100 \mathrm{PeV}$ for different core distances $R$. Generally, optimum $r_{i j}$ depends on the pair, $E_{0}$ and $R$. In each case the borders between criterion class distributions are adjusted so that misclassification errors (e.g., $P\{p \rightarrow N\}$ and $P\{N \rightarrow p\}$ ) are equal. Thus, Table 4 shows only one value. Errors for pure $C L$ signal are slightly lower than for $C L+B G$. Analysis shows that integration intervals 
Table 4: Misclassification probabilities for p-N and N-Fe pairs, $E_{0}=1,10,100 \mathrm{PeV}$. 1 and $10 \mathrm{PeV}$ samples contain 240 images, $100 \mathrm{PeV}$ samples - 120 images, QGSJET01 only

\begin{tabular}{|c|c|c|c|c|c|c|c|c|c|c|c|c|}
\hline$E_{0}$ & \multicolumn{4}{|c|}{$1 \mathrm{PeV}$} & \multicolumn{4}{|c|}{$10 \mathrm{PeV}$} & \multicolumn{4}{|c|}{$100 \mathrm{PeV}$} \\
\hline$R, \mathrm{~m}$ & 50 & 100 & 150 & 200 & 50 & 100 & 150 & 200 & 50 & 100 & 150 & 200 \\
\hline pair & \multicolumn{12}{|c|}{ signal: CL, ph, } \\
\hline $\mathrm{p}-\mathrm{N}$ & 0.05 & 0.10 & 0.15 & 0.17 & 0.09 & 0.13 & 0.17 & 0.21 & 0.12 & 0.13 & 0.17 & 0.23 \\
\hline $\mathrm{N}-\mathrm{Fe}$ & 0.05 & 0.10 & 0.15 & 0.15 & 0.004 & 0.04 & 0.09 & 0.12 & 0.02 & 0.05 & 0.08 & 0.11 \\
\hline pair & \multicolumn{12}{|c|}{ signal: CL+BG, phel, } \\
\hline $\mathrm{p}-\mathrm{N}$ & 0.05 & 0.11 & 0.15 & 0.17 & 0.09 & 0.13 & 0.17 & 0.20 & 0.11 & 0.13 & 0.17 & 0.23 \\
\hline $\mathrm{N}-\mathrm{Fe}$ & 0.05 & 0.10 & 0.15 & 0.16 & 0.01 & 0.04 & 0.09 & 0.13 & 0.02 & 0.05 & 0.08 & 0.11 \\
\hline pair & \multicolumn{12}{|c|}{ signal: CL, ph, } \\
\hline $\mathrm{p}-\mathrm{N}$ & 0.06 & 0.10 & 0.16 & 0.18 & 0.09 & 0.13 & 0.18 & 0.21 & 0.15 & 0.16 & 0.18 & 0.28 \\
\hline $\mathrm{N}-\mathrm{Fe}$ & 0.05 & 0.11 & 0.15 & 0.16 & 0.004 & 0.05 & 0.10 & 0.12 & 0.04 & 0.08 & 0.11 & 0.15 \\
\hline pair & \multicolumn{12}{|c|}{ signal: CL+BG, phel, } \\
\hline p-N & 0.05 & 0.11 & 0.15 & 0.17 & 0.09 & 0.13 & 0.17 & 0.20 & 0.16 & 0.16 & 0.23 & 0.28 \\
\hline $\mathrm{N}-\mathrm{Fe}$ & 0.05 & 0.11 & 0.17 & 0.16 & 0.01 & 0.05 & 0.10 & 0.13 & 0.03 & 0.07 & 0.12 & 0.15 \\
\hline
\end{tabular}

Table 5: Misclassification probabilities for $\mathrm{p}-\mathrm{N}$ and N-Fe pairs, QGSJET01 vs. QGSJET-II-04, $\quad E_{0}=1 \mathrm{PeV}$. All samples contain 240 images. Signal: CL+BG, phel, criterion: universal

\begin{tabular}{l|lllll|lllll}
\hline model & \multicolumn{5}{|c|}{ QGSJET01 } & \multicolumn{5}{c}{ QGSJET-II-04 } \\
\hline$R, \mathrm{~m}$ & 21 & 50 & 100 & 150 & 200 & 21 & 50 & 100 & 150 & 200 \\
\hline $\mathrm{p}-\mathrm{N}$ & 0.03 & 0.05 & 0.11 & 0.15 & 0.17 & 0.05 & 0.05 & 0.12 & 0.19 & 0.19 \\
$\mathrm{~N}-\mathrm{Fe}$ & 0.05 & 0.05 & 0.11 & 0.17 & 0.16 & 0.06 & 0.05 & 0.16 & 0.18 & 0.20 \\
\hline
\end{tabular}

Table 6: Probabilities $P\{p \rightarrow \gamma\} / P\{\gamma \rightarrow p\}$. All samples contain 400 images

\begin{tabular}{l|l|l}
\hline Pair & $50 \mathrm{~m}$ & $100 \mathrm{~m}$ \\
\hline $30 \mathrm{TeV} \gamma-60 \mathrm{TeV} \mathrm{p}$ & $0.0025 / 0.345$ & $0.0025 / 0.5125$ \\
$50 \mathrm{TeV} \gamma-100 \mathrm{TeV} \mathrm{p}$ & $0.0025 / 0.255$ & $0.0025 / 0.545$ \\
\hline
\end{tabular}

along the spot long axis, corresponding to optimum $r_{i j}$, mostly depend on $R$ and a universal feature $r=r(R)$ can be defined behaving almost optimally.

We also tested the performance of the universal criterion developed for QGSJET01 model with 1 PeV QGSJET-II-04 data. The comparison of the two models is summarized in Table 5.

Figure 3 presents integration limits for the universal feature as functions of $R$.

The introduced criterion exhibits superior sensitivity to the primary mass compared with $X_{\max }$ which is illustrated by figure 4 .

The same approach to CL images processing is capable of suppressing at least $99 \%$ of nuclear background in 30-100 $\mathrm{TeV} \gamma$-ray detection. In this case borders between $\gamma$ and proton classes are placed to leave only one proton in the $\gamma$ domain. Best results for $\mathrm{CL}+\mathrm{BG}$ turned into photoelectrons are shown in Table 6. 


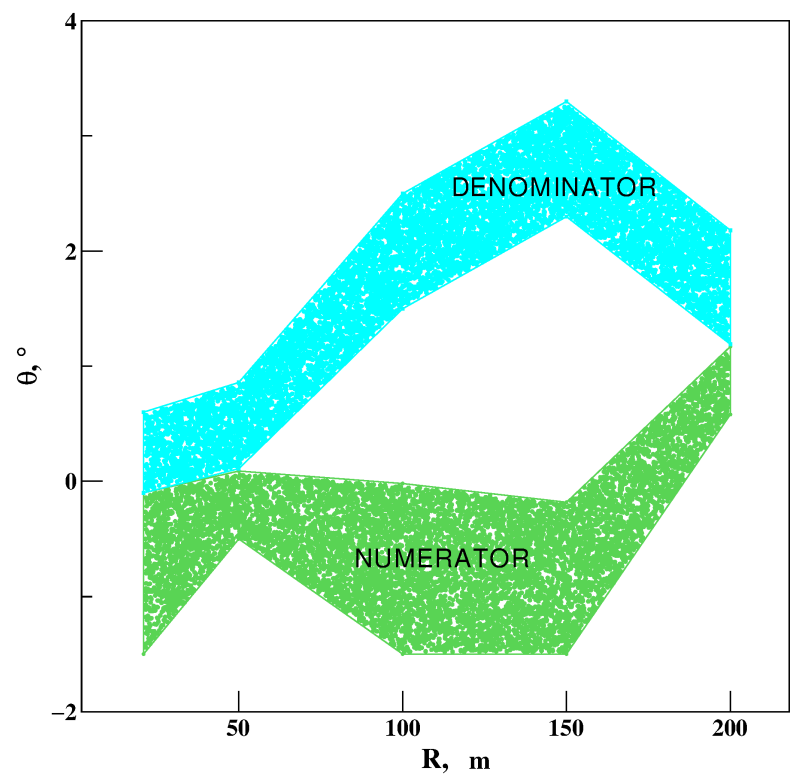

Figure 4: Showers with $X_{\max }=500 \mathrm{~g} / \mathrm{cm}^{2}$ initiated by $1 \mathrm{PeV}$ protons and nitrogen nuclei are separated by universal criterion at $R=50 \mathrm{~m}$
Figure 3: Integration limits for universal feature $r$ vs. $R$. Lower area: numerator integral. Upper area: denominator integral

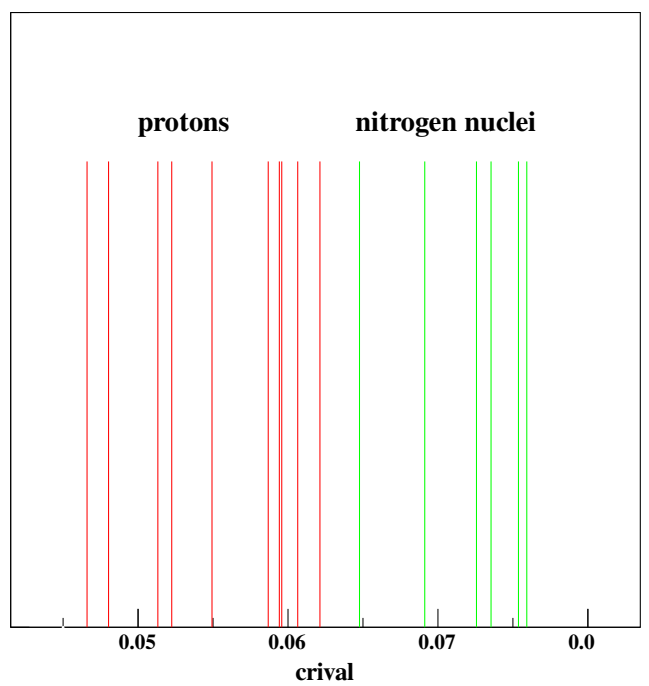

\section{Charged particle detector network}

We consider a fragment of charged particle detector network of $5 \times 5$ detectors $1 \mathrm{~m} \times 1 \mathrm{~m}$ each in order to optimize the spacing (grid step) and find the lower limits of uncertainties in the core location, arrival direction and primary energy estimation. Two versions of particle spatial-temporal distribution processing are considered:

a) according to CORSIKA particle data, the number of charged particles and the moment of particle front arrival in each detector are defined; using these "measured" data the core location and arrival direction are estimated as well as particle lateral distribution function which is then integrated to 
give a $E_{0}$ estimate;

b) all CORSIKA particles within a detector are tracked through its body ( $2 \mathrm{~mm}$ of $\mathrm{Al}+2 \mathrm{~cm}$ of polystyrene) with GEANT4 [3], by the deposited energies and centers of mass of ionization pulses the same event parameters as in a) are estimated.

Table 7: Uncertainties of primary direction $\delta_{d i r}$, core location $\delta_{l o c}$ and primary energy $\delta_{E_{0}}$ estimates by a network of $5 \times 5$ charged particle detectors for EAS produced by nearly vertical 1 and $10 \mathrm{PeV}$ protons

\begin{tabular}{|l|l|l|l|l|l|l|}
\hline case & \multicolumn{3}{|c|}{$\mathrm{a})$} & \multicolumn{3}{c|}{ b) } \\
\hline$E_{0}, \mathrm{PeV}$ & $\delta_{\text {dir }},{ }^{\circ}$ & $\delta_{\text {core }}, \mathrm{m}$ & $\delta_{E_{0}}, \%$ & $\delta_{\text {dir }},{ }^{\circ}$ & $\delta_{\text {core }}, \mathrm{m}$ & $\delta_{E_{0}}, \%$ \\
\hline 1 & 0.41 & 1.1 & 19 & 0.51 & 1.0 & 16 \\
\hline 10 & 0.15 & 0.6 & 12 & 0.18 & 0.3 & 12 \\
\hline
\end{tabular}

1 and $10 \mathrm{PeV}$ proton showers are used. In case a) 120 and 60 events are considered, in case b) 50 and 10 events, accordingly. In both cases each event is used many (1600) times. Three grid steps are tried: 10,15 and $20 \mathrm{~m}$. The shower axis is kept within the detector network fragment.

Step $15 \mathrm{~m}$ shows the best overall results in both cases (Table 7). $E_{0}$ is estimated by the integral of particle $F_{L D}(R)$ within a circle of $100 \mathrm{~m}$ radius in case a) or of ionization $F_{L D}(R)$ within the same radius as in case $b$ ).

\section{Conclusions}

1. The proposed optical part of Pamir-XXI is capable of achieving the required accuracies in core location $(\sim 1 \mathrm{~m})$, arrival direction (better than $\left.0.1^{\circ}\right)$ and primary energy $(\lesssim 15 \%)$ within the target primary energy range $30 \mathrm{TeV}-100 \mathrm{PeV}$. Using this detector array it is also possible to divide all primary nuclei into three groups $(1-100 \mathrm{PeV})$ and reject not less than $99 \%$ of nuclear background events while selecting $\gamma$-events $(30-100 \mathrm{TeV})$. Our criterion for the primary nuclei separation shows weak dependence on the interaction model.

2. The charged particle network can estimate the core location within $\sim 1 \mathrm{~m}$ accuracy, arrival direction with uncertainty of $\sim 0.5^{\circ}$ even at $1 \mathrm{PeV}$ and primary energy with uncertainty better than $20 \%$ in $1-10 \mathrm{PeV}$ energy range. A preferable grid step is $15 \mathrm{~m}$.

3. The question of whether and how this is possible to estimate the primary mass using charged particle detector data is still open. Probably, it is possible with muon trackers and/or central calorimeter with high spatial resolution.

\section{References}

[1] A. S. Borisov and V. I. Galkin, Journal of Physics: Conference Series, 409, No.1, 012089 (2013).

[2] D. Heck and T. Pierog Extensive Air Shower Simulation with CORSIKA: A User's Guide, (KARLSRUHER INSTITUT FUR TECHNOLOGIE, 2011).

[3] GEANT4 Collaboration. GEANT4 User's Guide for Application Developers. Version 10.1, 2014. 